\title{
Numerical integration of KPZ equation with restrictions
}

\author{
M F Torres $^{1,2}$ and R C Buceta ${ }^{1,2}$ \\ ${ }^{1}$ Instituto de Investigaciones Físicas de Mar del Plata, UNMdP and CONICET, \\ Funes 3350, B7602AYL Mar del Plata, Argentina \\ 2 Departamento de Física, FCEyN, Universidad Nacional de Mar del Plata \\ Funes 3350, B7602AYL Mar del Plata, Argentina \\ E-mail: mtorres@ifimar-conicet.gob.ar and rbuceta@mdp.edu.ar
}

Received 28 November 2017

Accepted for publication 20 February 2018

Published 20 March 2018

Online at stacks.iop.org/JSTAT/2018/033208

https://doi.org/10.1088/1742-5468/aab1b3

\begin{abstract}
In this paper, we introduce a novel integration method of KardarParisi-Zhang (KPZ) equation. It is known that if during the discrete integration of the KPZ equation the nearest-neighbor height-difference exceeds a critical value, instabilities appear and the integration diverges. One way to avoid these instabilities is to replace the KPZ nonlinear-term by a function of the same term that depends on a single adjustable parameter which is able to control pillars or grooves growing on the interface. Here, we propose a different integration method which consists of directly limiting the value taken by the KPZ nonlinearity, thereby imposing a restriction rule that is applied in each integration timestep, as if it were the growth rule of a restricted discrete model, e.g. restrictedsolid-on-solid (RSOS). Taking the discrete KPZ equation with restrictions to its dimensionless version, the integration depends on three parameters: the coupling constant $g$, the inverse of the time-step $k$, and the restriction constant $\varepsilon$ which is chosen to eliminate divergences while keeping all the properties of the continuous KPZ equation. We study in detail the conditions in the parameters' space that avoid divergences in the 1-dimensional integration and reproduce the scaling properties of the continuous KPZ with a particular parameter set. We apply the tested methodology to the $d$-dimensional case $(d=3,4)$ with the purpose of obtaining the growth exponent $\beta$, by establishing the conditions of the coupling constant $g$ under which we recover known values reached by other authors, particularly for the RSOS model. This method allows us to infer that
\end{abstract}


$d=4$ is not the critical dimension of the KPZ universality class, where the strong-coupling phase disappears.

Keywords: growth processes, interfaces in random media, kinetic roughening, classical Monte Carlo simulations

\section{Contents}

1. Introduction

2. Model and definitions

3. Results of the KPZ integration by restricting method 6

3.1. Results for 1-dimension ....................................................................... 6

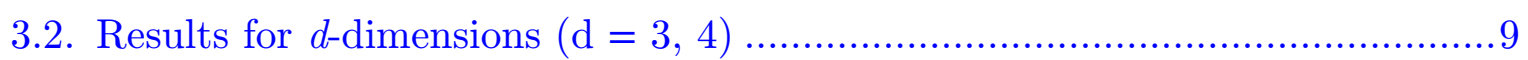

4. Conclusions 11

Acknowledgments ............................................................. 12

$\begin{array}{ll}\text { References } & 12\end{array}$

\section{Introduction}

Throughout the last three decades, intense research on models and equations has been done to understand the phenomenon of surface growth. Among the systems that grow out of equilibrium, perhaps the most studied ones are those included in the KardarParisi-Zhang (KPZ) universality class characterized by the homonymous stochastic equation [1]

$$
\frac{\partial h}{\partial t}=\nu \nabla^{2} h+\frac{\lambda}{2}(\nabla h)^{2}+\eta(\mathbf{x}, t)
$$

where $h=h(\mathbf{x}, t)$ is the surface height of a growing medium on a $d$-dimensional substratum, at position $\mathbf{x}$ and time $t$. The Laplacian term and the non-linear term represent the elasticity and the lateral growth of the interface, respectively. The noise $\eta(\mathbf{x}, t)$ is Gaussian with zero mean and covariance $\left\langle\eta(\mathbf{x}, t) \eta\left(\mathbf{x}^{\prime}, t^{\prime}\right)\right\rangle=2 D \delta^{d}\left(\mathbf{x}-\mathbf{x}^{\prime}\right) \delta\left(t-t^{\prime}\right)$, where $D$ is the noise intensity. The right-hand side of the KPZ equation (1) may include an additive term representing a constant force $f$ due to incoming or outgoing particle-flow that is absorbed or desorbed on the surface, respectively. For $\lambda=0$ the equation (1) becomes the Edward-Wilkinson (EW) equation [2]. A large number of real growing interfaces has been successfully described by the KPZ equation in one and two dimensions [3-6]. Growing lattice models with the same interface properties as the KPZ equation, e.g. ballistic deposition [7], restrictedsolid-on-solid (RSOS) [8, 9], etching algorithm [10], or Eden model [11], are also used as a substitute for addressing open questions. The existence of an upper critical dimension $d_{\mathrm{u}}$ above of which fluctuations are negligible, independently of the 
KPZ nonlinearity strength, is currently the most salient unresolved issue [12-36]. In order to consider that the KPZ equation is a proper hydrodynamic description of the interface growth of some real system or growth model, they both need to have the same scaling properties and exponents. The KPZ equation has Galilean invariance, regardless of the dimension $d$, if the relation $\zeta+z=2$ is verified $[1,3$, 37 , where $\zeta$ is the (global) roughness exponent and $z$ is the dynamical exponent. For $d=1$, since the system behaviour verifies the fluctuation-dissipation theorem [1], we know that $\zeta=1 / 2$. This theorem is not valid for $d>1$ for which the exponents must be calculated in a different way; however, an analytical method that allows to know the exponents or scaling properties has not been established yet. Several numerical methods have been developed with similar results in some cases and dissimilar in others. The methods of perturbative renormalization $[1,3,12,13]$, with coupling constant $g=\lambda^{2} D / \nu^{3}$, predict that for every dimension $d>2$ there is a critical value $g_{\mathrm{c}} \neq 0$ that separates two regions: for $g<g_{\mathrm{c}}$ a weak-coupling phase with $\zeta=0$ and for $g>g_{\mathrm{c}}$ a strong-coupling phase with $\zeta \neq 0$. However, these methods have been unable to obtain exponents in the strong-coupling phase. On the one hand, studies of the mapping of the KPZ equation in the directed polymers with quenched noise [14, 15] and self-consistent methods [16-21] predict a critical dimension $d_{\mathrm{u}} \leqslant 4$, where the strong-coupling phase disappears. On the other hand, by using a non-perturbative renormalization method in the real space, exponents of the strong-coupling phase up to dimension $d=9$ were obtained, without the prediction of a critical dimension [22-24]. In addition, these exponents are close to those obtained from the simulation of growth models, which share the properties of KPZ equation exactly at $d=1$. Specifically, the RSOS model has been simulated up to dimension 11, without showing signs of a critical dimension [8-10, 25-32]. Furthermore, large simulations of a directed polymer model on random potential at finite temperature in $d=4[35,36]$ also contradict $d_{\mathrm{u}} \leqslant 4$, which was established analytically by Lässig and Kinzelbach [15]. The discrete integration of the KPZ equation is another of the methods used to obtain its scaling properties. The KPZ equation is usually numerically integrated following a discretization scheme like this [38]:

$$
h_{j}(t+\Delta t)=h_{j}(t)+\sum_{i=1}^{i=d}\left(\nu L_{j}^{[i]}+\frac{\lambda}{2} N_{j}^{[i]}\right) \Delta t+\sigma \sqrt{12 \Delta t} R_{j}(t),
$$

where $\Delta t$ is the integration time-step and $h_{j}$ is the height of interface on the $j$ th lattice point. The noise amplitude is $\sigma \sqrt{12 \Delta t}$, where $\sigma=\sqrt{2 D /(\Delta x)^{d}}$, and $R(t)$ is a uniform random variable between $[-0.5,0.5]$. The linear term $L_{j}^{[i]}=\left(h_{j+1}^{[i]}-2 h_{j}+h_{j-1}^{[i]}\right) /(\Delta x)^{2}$ is the discrete $\partial^{2} h / \partial x_{i}^{2}$ of the Laplacian, where $\Delta x$ is the integration mesh-step and $h_{j \pm 1}^{[i]}$ are the heights of nearest-neighbours (NN) of the $j$ th lattice point in the $i$ th direction. The nonlinear term of the KPZ can be discretized by adopting different schemes that are equivalent [39], although the simplest one is the Euler scheme, in which the nonlinear term of equation (2) is $N_{j}^{[i]}=\left[\left(h_{j+1}^{[i]}-h_{j-1}^{[i]}\right) /(2 \Delta x)\right]^{2}$. Replacing $h_{j}=h_{0} H_{j}$, $x=x_{0} r$ and $t=t_{0} \tau$ in equation (2) we obtain the dimensionless equation 


$$
H_{j}(\tau+\Delta \tau)=H_{j}(\tau)+\sum_{i=1}^{i=d}\left(\mathcal{L}_{j}^{[i]}+\frac{1}{2} \mathcal{N}_{j}^{[i]}\right) \Delta \tau+\sqrt{12 \Delta \tau} R_{j}(\tau)
$$

where $h_{0}=\nu / \lambda, x_{0}=\sqrt{\nu^{3} /(\sigma \lambda)^{2}}$, and $t_{0}=[\nu /(\sigma \lambda)]^{2}$. In the last equation, the linear and nonlinear terms are

$$
\begin{aligned}
\mathcal{L}_{j}^{[i]} & =\frac{H_{j+1}^{[i]}(\tau)-2 H_{j}(\tau)+H_{j-1}^{[i]}(\tau)}{(\Delta r)^{2}}, \\
\mathcal{N}_{j}^{[i]} & =\left[\frac{H_{j+1}^{[i]}(\tau)-H_{j-1}^{[i]}(\tau)}{2 \Delta r}\right]^{2},
\end{aligned}
$$

respectively.

The KPZ discrete integration presents instabilities that make its simulation diverge quickly [40, 41]. A deep analysis made by Dasgupta et al [42, 43] shows that these instabilities are caused by the uncontrollable growth of pillar or grooves that are intrinsic to discrete versions of equations with nonlinear terms $(\nabla h)^{2}$, with or without noise. Instabilities cannot be avoided by enlarging the system or using a generalized discretization of the nonlinear term; not even by reducing the integration time step, which only makes their appearance less probable. The instability in the numerical integration of the one-dimensional KPZ equation with $\lambda>0(\lambda<0)$ is associated with grooves (pillars). Dasgupta et al showed that a critical height $h_{\mathrm{c}} \propto-\lambda^{-1}$ exists using numerical integration starting from a flat interface with a pillar or groove perturbation of height $h_{\mathrm{p}}$ at some point in the mesh. Below the critical value (i.e. $\left|h_{\mathrm{p}}\right|<\left|h_{\mathrm{c}}\right|$ ), the perturbation is reabsorbed into the interface, and above it, the interface diverges quickly. It can be observed that if during the discrete integration of the KPZ equation the NN height difference surpasses a critical value, the instability appears and the integration diverges. The exact solution to the one-dimensional KPZ equation found by Sasamoto and Spohn [44] shows that there are no instabilities in the continuous version. Additionally, the noiseless continuous KPZ equation can be mapped into a diffusion equation by a ColeHopf transformation and be exactly solved without instabilities. Conversely, the application of such a transformation to the noiseless discrete KPZ equation is not reduced to a discrete diffusion equation, which suggests a possible explanation for the generation of instabilities along the numerical integration. To successfully integrate the KPZ, Dasgupta et al $[42,43]$ propose to replace the non-linear term $(\nabla h)^{2}$ with a function $\Phi\left((\nabla h)^{2}\right)$ in the KPZ equation, defined by $\Phi(y)=\left(1-\mathrm{e}^{-c y}\right) / c$, where $c$ is an adjustable constant. This proposal does not require a special discretization scheme, since it is possible to maintain the Euler scheme without loss of generality. This method avoids, within a certain range of the parameter $c$, the big local height-differences that lead to excessive growth, which are the origin of instabilities. Introducing the nonlinear function $f$ into the KPZ equation, which is equivalent to introducing an infinite nonlinear series, must leave the scaling properties of universal quantities invariant and eliminate divergences. Some properties of the KPZ equation, such as scaling exponents, can be calculated with this procedure [43] with great precision and coinciding with the 
theoretical values from renormalization group for $d=1$ and growth models for $d=2$ [45]. If $c \gg 1$, nonlinear effects become weak and, in the extreme case, a long transient with scaling properties of the EW universality class appears. On the contrary, if $c \ll 1$, the method fails to avoid instabilities.

In this work, we propose a different integration model inspired by the study of the instabilities made by Dasgupta et al [43]. By our method, we directly limit the value of the non-linear term, restricting the value of height-difference between NN columns, a difference which is responsible for making integration divergent. In this paper, we characterize this method and use it to regain and obtain important properties of the KPZ equation. In section 2, we introduce and characterize the restricted integration method. In section 3.1, we show the integration properties of the KPZ equation in $d=1$ and obtain the associated exponents. In section 3.2, we show the results for $d=3$, which were never obtained with an integration scheme that avoids divergences; and for $d=4$, which are of great importance since the KPZ numerical integration was never performed until this dimension, and because it is the dimension that several authors predict to be critic, i.e. that does not present a roughness region.

\section{Model and definitions}

To integrate the KPZ equation we are going to use a similar scheme to the one presented in equation (3), but with an upper limit to nonlinearity $\mathcal{N}_{j}^{[i]}$ given by equation (5). Our proposal is to replace this term as follows:

$$
\mathcal{N}_{j}^{[i]} \longrightarrow \frac{1}{(\Delta r)^{2}}\left[\left(\mathcal{N}_{j}^{[i]}(\Delta r)^{2}-\varepsilon\right) \Theta\left(\varepsilon-\mathcal{N}_{j}^{[i]}(\Delta r)^{2}\right)+\varepsilon\right],
$$

where $\Theta(x)$ is the Heaviside step function. The restricted growth rule $\left|H_{j+1}^{[i]}(\tau)-H_{j-1}^{[i]}(\tau)\right| \leqslant 2 \sqrt{\varepsilon}$ applies for each integration time-step, as if it were the growth rule of a discrete model with restrictions (e.g. RSOS). The restriction constant $\varepsilon$ must be chosen in order to eliminate the divergences that arise in the usual integration of the discrete KPZ equation and, at the same time, to maintain all the basic properties of the continuous KPZ equation. From equation (3) we can see that $\varepsilon$ depends on the integration steps $\Delta r$ and $\Delta \tau$. By means of the coupling constant $g=\lambda^{2} D / \nu^{3}=\lambda^{2} \sigma^{2} / 2 \nu^{3}$, introduced from the KPZ studies by renormalization group theory, it is easy to see that integration depends on the coefficients and the integration steps through equations $\Delta r=\sqrt{\frac{2 g}{\Delta x^{(d-2)}}}$ and $\Delta \tau=\frac{2 \nu g}{\Delta x^{d}} \Delta t$. Notice that the integration steps $\Delta r$ and $\Delta \tau$ are invariant under the transformations $\Delta x \rightarrow 1, \Delta t \rightarrow \Delta t /(\Delta x)^{2}$ and $g \rightarrow g /(\Delta x)^{d-2}$. This property allows us to take $\Delta x=1$ without loss of generality. In addition, by choosing $\Delta t=1 /(2 k \nu g)$ (where $k$ is a positive real constant) we make the integration simpler by ensuring the same statistic for each $g$ value, $\Delta \tau=1 / k$. In short, the integration depends on only three parameters: the coupling constant $g$, the inverse of time step $k$, and the restriction value $\varepsilon$. 
In order to obtain the range of $\varepsilon$ values for which the properties of the KPZ equation remain invariant, we study the probability that the restriction to growth occurs, i.e. $\Psi(\varepsilon)=P(X>\varepsilon)=\langle\Theta(X-\varepsilon)\rangle$, where $X=\left\{\frac{1}{2}\left[H_{j+1}^{[i]}(\tau)-H_{j-1}^{[i]}(\tau)\right]\right\}^{2}$.

One of the main observables that can be measured in a growing interface to characterize its evolution is its width or roughness defined by $w(\tau)=\left[\left\{\langle h(\mathbf{r}, \tau)\rangle^{2}-\left\langle h(r, \tau)^{2}\right\rangle\right\}\right]^{1 / 2}$, where $\langle\cdots\rangle$ is the average over the interface of size $L$ and $\{\cdots\}$ over the different realizations. Usually, the system has a power law behaviour with $w \propto \tau^{\beta}$ for $\tau \ll \tau_{x}$, where $\beta=\zeta / z$ is the growth exponent, and that the system saturates with $w=w_{\text {sat }} \propto L^{\zeta}$ for $\tau \gg \tau_{\mathrm{x}}$. Also, it has been found that the crossover time $\tau_{\mathrm{x}} \propto L^{z}$. In the case of the KPZ equation, because of its Galilean invariance, it is known that $\zeta=1 / 2$ and $z=3 / 2$ for $d=1$ and $z+\beta=2$ for any dimension $d$.

Another observable measured in a growing interface is the height-difference correlation of $m$ th-order defined by $G_{m}(\ell, \tau)=\left\{\left\langle\left|H_{j+\ell}^{[i]}(\tau)-H_{j}^{[i]}(\tau)\right|^{m}\right\rangle\right\}$, where $\ell$ is the distance between two columns. For growing systems, this correlation will either present a power law behaviour $G_{m} \propto \ell^{m \zeta_{m}}$ for $\ell \ll \xi(\tau)$ or take a constant value $G_{m}^{\text {sat }}$ for $\ell \gg \xi(\tau)$, where $\xi(\tau)$ is the correlation length. Also, $\xi \propto \tau^{1 / z}$ for $\tau<\tau_{\times}$and equal to the maximum neighbour distance for $\tau>\tau_{\mathrm{x}}$ (e.g. $\xi=L / 2$ for systems with periodic boundary conditions). When $\zeta_{m}$ depends on $m$ the correlation shows multiscaling and the system is multi-affine. Otherwise, the correlation shows single scaling and the system is self-affine. The particular case $m=2$ allows to relate the correlation with the roughness. It can be noted that $G_{2} \propto \tau^{2\left(\zeta-\zeta_{2}\right) / z} \ell^{2 \zeta_{2}}$ for $\ell \ll \xi$, where $\zeta_{2}$ is the local roughness exponent, and $G_{2}=G_{2}^{\text {sat }} \propto \tau^{2 \beta}$ for $\ell \gg \xi$. When $\zeta=\zeta_{2}$, the interface has usual or Family-Vicsek scaling, and in other cases, it has anomalous scaling.

\section{Results of the KPZ integration by restricting method}

\subsection{Results for 1-dimension}

We begin by analyzing the results of the simulations with the dimensionless KPZ equation in 1-dimension. We use Euler (or pre-point) discretization and growth restrictions by establishing a maximum height difference around the evolving site. Figure 1 shows the plot of probability $\Psi$ as a function of $\varepsilon$ for several values of $k=(\Delta \tau)^{-1}$ and $g=12.56 \approx 4 \pi$. We chose this value because it was originally reported by Moser et al that in a non-dimensionless and non-restricted integration it is the best value for reproducing the KPZ exponents [38]. The probability $\Psi$ shows two well differentiated behaviours as a function of the inverse time step $k$.

For $k \leqslant k^{*}$, the function $\Psi$ quickly decreases with $\varepsilon$ to a minimum value at $\varepsilon=\varepsilon^{*}(k)$ and then rapidly increases, where $\varepsilon^{*}$ is a monotonically increasing function of $k$. The minimum of $\Psi$ is visually on the plot for values of $k<k^{*} \approx 2$. This particular behaviour of $\Psi$ can be understood by plotting the height-difference correlation of 2nd-order at the saturation $G_{2}^{\text {sat }}$ as a function of time $\tau$, for different $\epsilon$ values, as shown in figure 2 for $k=1.5$ and $g=12.56$. Note that $G_{2}^{\text {sat }}$ shows the same behaviour as the roughness $W$, both as a function of time $\tau$, for the KPZ equation. For $\varepsilon=\varepsilon^{*}$, a power law behaviour of the correlation $G_{2}^{\text {sat }}$ with growth exponent very close to the theoretical value 
of the KPZ growth exponent ( $\beta_{\mathrm{KPZ}}=1 / 3$ ) is observed. For $\varepsilon<\varepsilon^{*}$ the power law holds although the exponent moves away as we move away. For $\varepsilon \ll \varepsilon^{*}$ we can observe that the measured exponent approaches the theoretical value of the EW growth exponent $\left(\beta_{\mathrm{EW}}=1 / 4\right)$. This change in behaviour is a consequence of the nonlinear term being strongly restricted and diffusion being dominant. Conversely, for $\varepsilon>\varepsilon^{*}$, the behaviour of the power law is temporarily shortened with an exponent close to the theoretical value in the early- and middle-time regime and, later, it shows a divergent behaviour. This is accentuated when the $\varepsilon$ value is far from $\varepsilon^{*}$; the $\varepsilon$ value is so large that it is unable to limit divergences of integration. In this last case, the method becomes ineffective and presents the same difficulties as the usual integration method.

For $k>k^{*}$, no minimum value is observed for the probability $\Psi(\varepsilon)$, only that it always decreases as a function of $\varepsilon$, becoming very small or zero when $\varepsilon$ increases, which assures us that the restriction is rarely or never applied. Even so, the restriction must be maintained, since a divergence in merely one sample would destroy the average. For $k>k^{*}$ it suffices to stay in the last value of $\varepsilon$ for which $\Psi \neq 0$. Otherwise, for $k=k^{*}$, we consider the value $\varepsilon=\varepsilon^{*}$ where $\Psi$ has a minimum.

To observe how the growth exponent $\beta$ depends on the coupling parameter $g$ we study the correlation $G_{2}^{\text {sat }}$ as a function of time $\tau$ for different values of $g$. Taking $L=512$ and $k=100$, in figure 3 we observe how the power laws are maximized in the neighbourhood of $g=15$. For values of $g \ll 15$, the elastic term causes a rapid saturation of the interface, producing a small deviation from the expected KPZ-value of $\beta$. For $g \gg 15$, the noise term affects the growth at the beginning, delaying the KPZ behaviour. Both behaviours are due to finite-size effects, i.e. for all $g$, as the system size grows, $\beta \rightarrow \beta_{\mathrm{KPZ}}$.

Results of our simulations show that the system is self-affine. The $m$ th-order correlation $G_{m}$ as a function of the distance $\ell$ between columns shows that $\zeta_{1} \approx 0.487$, $\zeta_{2} \approx 0.489, \zeta_{3} \approx 0.488$, and $\zeta_{4} \approx 0.489$ for a system of size $L=16384, k=10$ and and time $\tau=2^{20}$ close to the saturation. The exponent values in different orders are very close to each other, which confirms that the restrictions do not introduce changes to the results known for the KPZ. Simulations show that the system with restriction maintains the self-affinity for different sizes $L$ and for different values of the coupling constant $g$.

Plot (a) of figure 4 shows $G_{2}$ as a function of $\ell$, for different values of time $\tau$, size $L=16384$ and $k=10$. We observe that $\zeta_{2}$ depends on $\tau$ and it approaches the KPZ theoretical value $\left(\zeta_{\mathrm{KPZ}}=1 / 2\right)$ as the time $\tau$ increases. As other authors have shown [45], when the system size $L$ and time $\tau$ go $+\infty$ the global roughness exponent $\zeta_{2}(\tau) \rightarrow 1 / 2$. Since the correlation lenght $\xi \propto \tau^{1 / z}$ we plot in figure $4(\mathrm{~b})$ the scaling function $\ell^{-2 \zeta_{\mathrm{KPz}}} G_{2}$ as a function of $\ell \tau^{-1 / z_{\mathrm{KPZ}}}$ for several values of $\tau$. In the plot we can see that the interface has usual scaling, since the different curves overlap. It is important to note that, at distance $\ell_{0} \approx 2$, the correlation $G_{2}\left(\ell_{0}, \tau\right)$ is constant with time $\tau$, except for small initial variations (see figure 4). Therefore, if we increase the size of the system, bringing it to the thermodynamic limit, it is not necessary to modify the restriction that allows the integration. In contrast, as the system size increases, our method for growth models with anomalous scaling (e.g. LD equation) requires to modify the restriction that avoids instabilities. Similarly, the method of integration of Dasgupta et al for the LD equation shows that by increasing the size of the system it is necessary to modify the parameter $c$ that controls the nonlinearities which avoid instabilities [42, 43]. 


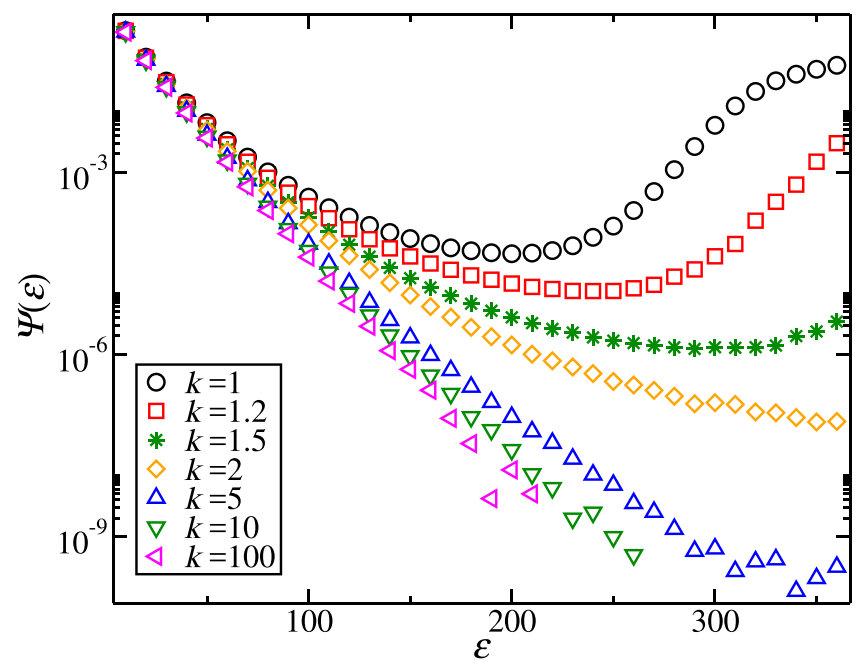

Figure 1. Plot of the probability $\Psi$ as a function of the restriction constant $\varepsilon$ for several values of $k=(\Delta \tau)^{-1}$, where $\Delta \tau$ is the integration time-step. We take $L=512$ and $g=12.56$. We use the integration mesh-step $\Delta r=\sqrt{2 g}$ corresponding to taking $\Delta x=1$.

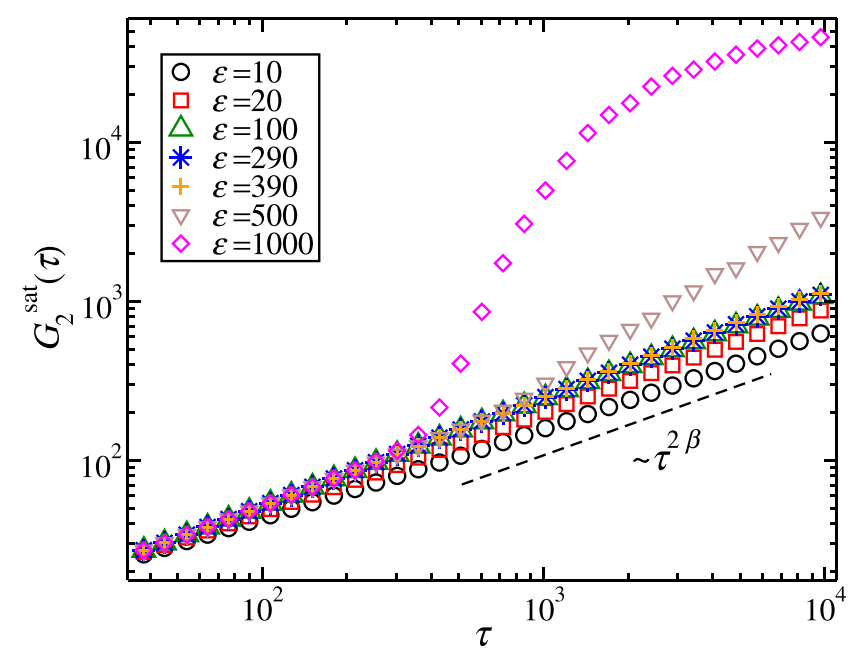

Figure 2. Height difference correlation of 2 nd-order at the saturation $G_{2}^{\text {sat }}$ as a function of the adimensional time $\tau$ for several values of $\varepsilon$. We use the same data of figure 1 and $k=1.5$. The measured values of the roughness exponent $\beta$ (in the cases where it can be measured) are $0.291(\varepsilon=10), 0.324(\varepsilon=20), 0.335(\varepsilon=100)$, $0.338(\varepsilon=290)$ and $0.344(\varepsilon=390)$. The plot shows for $\varepsilon=290 \cong \varepsilon^{*}(1.5)$ (blue upward-triangle) the power law behaviour of the correlation, for four decades, with growth exponent $\beta$ very close to the KPZ theoretical exponent $\beta_{\mathrm{KPZ}}=1 / 3$. Below, for $\varepsilon=10$ (black circles) the power law behaviour of the roughness exponent is close to the EW theoretical exponent $\beta_{\mathrm{EW}}=1 / 4$. Above, for $\varepsilon=500$ (orange lefttriangle) the power law behaviour, like KPZ, is lost over time due to divergences in integration. 


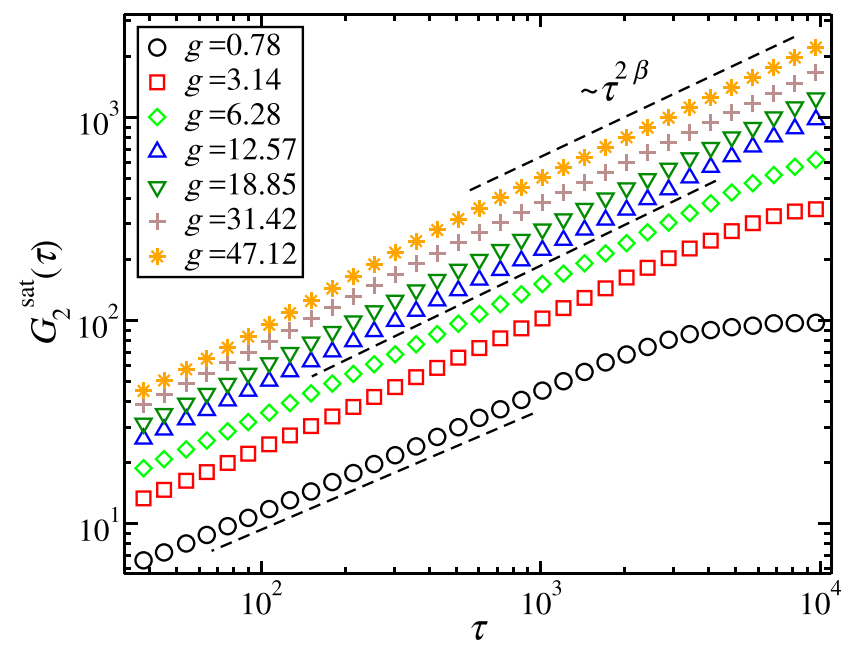

Figure 3. Saturation of the 2nd-order height-difference correlation $G_{2}^{\text {sat }}$ as a function of time $\tau$ for several values of $g$. For the simulations we used $L=512$, $k=100$ and $\varepsilon$ chosen as follows: $\varepsilon=200$ for $g<4 \pi$ and $g=300$ otherwise. The measured values of the roughness exponent $\beta$ are $0.298(g=0.78 \approx \pi / 4), 0.324$ $(g=3.14 \approx \pi), 0.330(g=6.28 \approx 2 \pi), 0.331(g=12.56 \approx 4 \pi), 0.333(g=18.85 \approx 6 \pi)$, $0.330(g=31.42 \approx 10 \pi)$ and $0.329(g=47.12 \approx 15 \pi)$.

Similar results are observed when the KPZ equation (1) includes an additive term representing a constant force $f \neq 0$ due to incoming or outgoing particle-flow. As $f$ increases, the only relevant change is an initial deformation in the roughness similar to incresing $g$ in figure 3 .

\subsection{Results for $d$-dimensions $(d=3,4)$}

By applying the methodology tested in the 1-dimensional case we analyse some important results that can be obtained in $d$-dimensions $(d>2)$, considering the cases $d=3,4$ in particular. For $d=3$ the height difference correlation of 2 nd order at the saturation $G_{2}^{\text {sat }}$ shows that there is a critical coupling value $g_{\mathrm{c}}$ that separates two well-differentiated behaviours. Below $\left(g<g_{\mathrm{c}}\right)$, the weak-coupling phase can be observed, where $\beta \approx 0$ and the system saturates rapidly. For $g \approx g_{\mathrm{c}}$, since it is a critical point, a power law-like behaviour is observed, with $\beta \gtrsim 0$. Above $\left(g>g_{\mathrm{c}}\right)$, whenever $g$ increases the interface roughness begins to increase as well. For a fixed value $g=g_{\mathrm{o}}$, the optimal power-law behaviour of the strong-coupling phase is obtained. For $g>g_{\mathrm{o}}$, as well as for $d=1$, it can be observed that the noise initially affects the power law, delaying the appearance of the KPZ type behaviour, but maintaining the exponents approximately. In figure 5(a), the correlation $G_{2}^{\text {sat }}$ is plotted as a time-dependent function $\tau$ for $d=3$ at different values of $g$. We observe that $g_{\mathrm{c}} \approx 30$ measuring $\beta(30) \approx 0.045$ and that the value of $g$ for which the best power law is obtained is close to $g_{\mathrm{o}} \approx 47$, with $\beta(47) \approx 0.204$. For higher values of $g$, the exponent value is $\beta \gtrsim 0.203$, except for $g=50$, where a greater deviation occurs, reaching $\beta(50) \approx 0.22$. We believe that this deviation occurs because the noise smoothly modifies the power law before transforming into the deformation seen for larger $g$ values. However, an exponent value $0.203 \lesssim \beta \lesssim 0.220$ is very close to the value measured for the RSOS model [25]. Taking $\beta=0.203$ and accepting the relation 

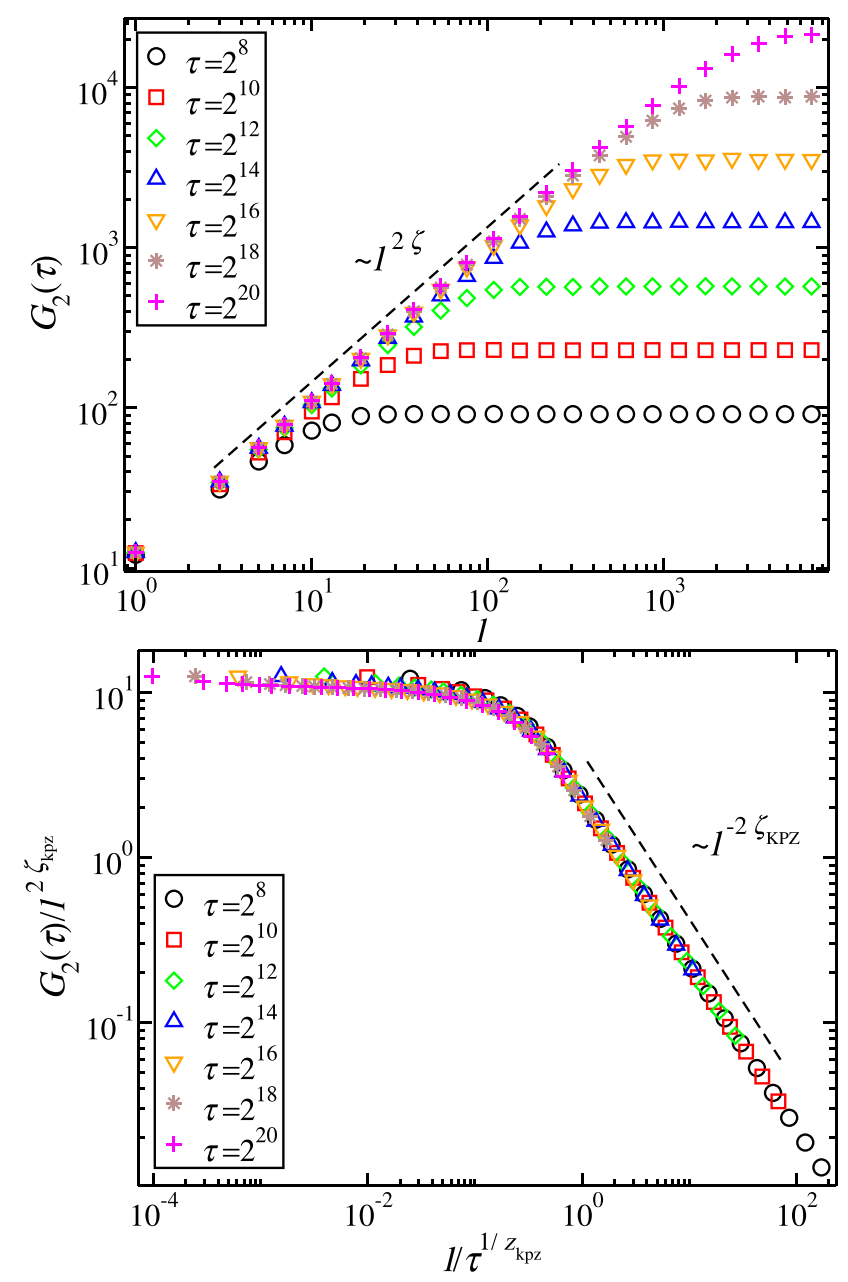

Figure 4. (a) Height-difference correlation of 2nd-order $G_{2}$ as a function of the distance between columns $\ell$ for several times $\tau=2^{n}$ (with $n$ integer), taking $L=16384, k=100, g=12.56$, and $\varepsilon=250$. The measured values of the local roughness exponent $\zeta_{2}$ are $0.440(n=10), 0.457(n=12), 0.471(n=14), 0.479$ $(n=16), 0.485(n=18)$ and $0.489(n=20)$. As $\tau$ increases $\zeta_{2}$ approaches the KPZ theoretical value of the global roughness exponent $\zeta_{\mathrm{KPZ}}=1 / 2$. (b) Scaling of the $G_{2}(\ell, \tau)$ for the same parameters and values of $\tau$ as those used in the plot (a). The plot shows $G_{2}=$ const for $\ell \ll \tau^{1 / z}$ and $G_{2} \propto \tau^{2 \beta}$ for $\ell \gg \tau^{1 / z}$.

$\zeta+z=2($ with $z=\zeta / \beta)$, we obtain $z \approx 1.662$ and $\zeta \approx 0.337$. For the RSOS model, the roughness exponent $\zeta \approx 0.313$ was measured as well [27], a value with a small departure from those mentioned above.

In the case of $d=4$, the correlation $G_{2}^{\text {sat }}$ is plotted as a function of time $\tau$ for different values of $g$ on the (b) of figure 5. A similar behaviour to the case $d=3$ can be observed, with $g_{\mathrm{c}} \approx 80$ and $g_{\mathrm{o}} \approx 100$, measuring $\beta(100) \approx 0.159$. This value is very close to the one measured for RSOS in the same dimension [30]. Taking the recently measured exponent $\zeta \approx 0.273$ for the RSOS model $[28,30]$, applying the relation $\zeta / \beta+\zeta=2$, we calculate $\beta \approx 0.158$, a value very close to the one reported here. For $g>100$, the measured values for the $\beta$ exponent begin to significantly deviate from the values accepted and retrieved here. Since the system size is small, for $d=4$ it saturates fast and we cannot conclude if the KPZ behaviour is recovered as it was for $d=1$ and $d=3$. 


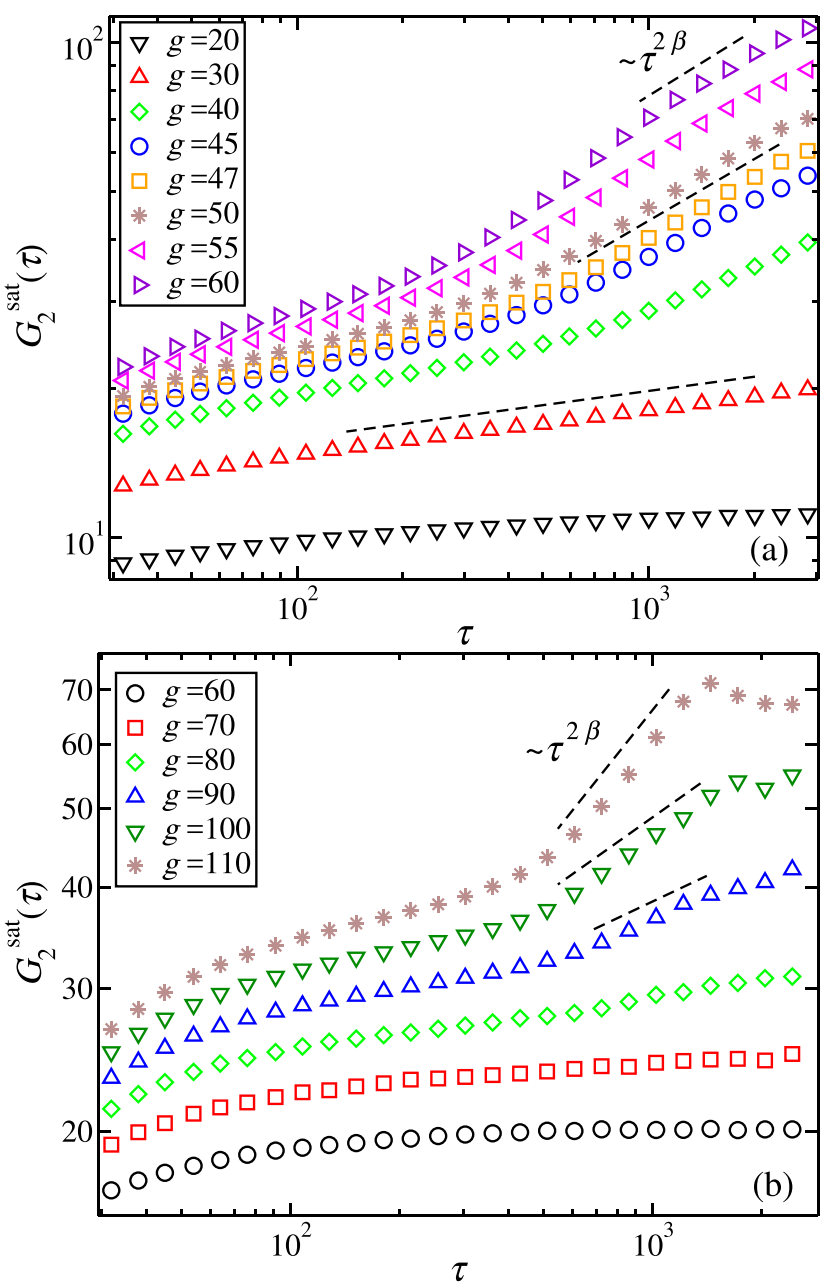

Figure 5. Both plots: Height-difference correlation of 2 nd-order $G_{2}^{\text {sat }}$ at the saturation as a function of the time $\tau$ for several $g$. For $d=3$ (Plot (a)) we take $L=60, k=50$ and $\varepsilon=130$. The measured values of the roughness exponent $\beta$ (in the cases where it can be measured) are $0.045(g=30), 0.156(g=40), 0.193$ $(g=45), 0.204(g=47), 0.220(g=50), 0.198(g=55)$ and $0.201(g=60)$. For $d=4$ (Plot (b)) we take $L=16, k=100$ and $\varepsilon=130$. The measured values of the roughness exponent $\beta$ (in the cases where it can be measured) are $0.106(g=90)$, $0.159(g=100)$ and $0.273(g=110)$.

\section{Conclusions}

In this paper, we demonstrate how it is possible to avoid the instabilities associated with the uncontrollable growth of pillars or wells that can be developed during the evolution of the discrete version of the KPZ equation. Contrary to the proposal introduced 20 years ago by Dasgupta et al, we propose not to modify the discrete KPZ equation by adding nonlinear terms, but to impose restrictions to the lateral growth of the interface by limiting the nonlinearities of the KPZ equation. The restriction rule is applied at each integration time step, but only acts to eliminate divergences while maintaining all the properties of the continuous KPZ equation. The $\varepsilon$ restriction parameter is chosen in a range of values that leaves the scaling properties invariant. In this work, we have 
integrated the discrete version of the dimensionless KPZ equation in such a way that it only depends on two parameters besides $\varepsilon$ : the inverse of the time-step $k$ and the coupling constant $g$. Our results in 1-dimension, with Euler discretization and growth restrictions, show that the height-difference correlation of 2 nd-order at the saturation is a power law with a growth exponent very close to the theoretical value established for the KPZ equation, i.e. $\beta_{K P Z}=1 / 3$. The method becomes effective for all times under these conditions. Otherwise, if $\varepsilon \ll \varepsilon^{*}$, the growth exponent is close to the theoretical value of the EW universality class $\left(\beta_{E W}=1 / 4\right)$, and if $\varepsilon \gg \varepsilon^{*}$, the KPZ power law can be broken due to the emergence of divergences with a probability that decreases with $k$. The method was tested for $d=3$ and $d=4$. In the first case, the predicted result is obtained by numerical methods, showing both the weak- and strong-coupling phases. Our results yield a critical-coupling constant $g_{\mathrm{c}} \approx 30$ and an optimal-coupling constant $g_{\mathrm{o}} \approx 47$ of the strong-coupling phase, for which the exponent $\beta$ is close to the one measured for RSOS simulations. When integrating for $d=4$, a strong-coupling phase is observed for $g>g_{\mathrm{c}} \approx 80$ with an exponent close to the one measured for RSOS at the optimal-coupling constant $g_{\mathrm{o}} \approx 100$. This can be taken as an indicator that $d=4$ is not the critical dimension of the KPZ universality class. Another option is that the observed strong-coupling phase is attributed to the finite size of the sytem. However, in our simulations, increasing the size of the system does not result in the disappearance of this phase.

\section{Acknowledgments}

This work was partially supported by Consejo Nacional de Investigaciones Científicas y Técnicas (CONICET), Argentina, PIP 2014/16 No. 112-201301-00629. RCB thanks

$\mathrm{C}$ Rabini for her suggestions on the final manuscript.

\section{References}

[1] Kardar M, Parisi G and Zhang Y-C 1986 Dynamic scaling of growing interfaces Phys. Rev. Lett. 56889

[2] Edwards S F and Wilkinson D R 1982 The surface statistics of a granular aggregate Proc. R. Soc. A 38117

[3] Barabasi A L and Stanley H E 1995 Fractal Concepts in Surface Growth (Cambridge: Cambridge University Press)

[4] Krug J 1997 Origins of scale invariance in growth processes Adv. Phys. 46139

[5] Krim J and Palasantzas G 1995 Experimental observations of self-affine scaling and kinetic roughening at sub-micron lengthscales Int. J. Mod. Phys. B 9599

[6] Miettinen L, Myllys M, Merikoski J and Timonen J 2005 Experimental determination of kpz heightfluctuation distributions Eur. Phys. J. B 4655

[7] Vold M J 1959 A numerical approach to the problem of sediment volume J. Colloid Sci. 14168

[8] Kim J M and Kosterlitz J M 1989 Growth in a restricted solid-on-solid model Phys. Rev. Lett. 622289

[9] Kim J M, Kosterlitz J M and Ala-Nissila T 1991 Surface growth and crossover behaviour in a restricted solid-on-solid model J. Phys. A: Math. Gen. 245569

[10] Mello B A, Chaves A S and Oliveira F A 2001 Discrete atomistic model to simulate etching of a crystalline solid Phys. Rev. E 63041113

[11] Eden M 1961 A two-dimensional growth process Proc. 4th Berkeley Symp. Mathematical Statistics and Probability vol 4 (Berkeley, CA: University of California Press) p 223

[12] Tang L-H, Nattermann T and Forrest B M 1990 Multicritical and crossover phenomena in surface growth Phys. Rev. Lett. 652422

[13] Frey E and Täuber U C 1994 Two-loop renormalization-group analysis of the Burgers-Kardar-Parisi-Zhang equation Phys. Rev. E 501024 
[14] Halpin-Healy T 1990 Disorder-induced roughening of diverse manifolds Phys. Rev. A 42711

[15] Lässig M and Kinzelbach H 1997 Upper critical dimension of the Kardar-Parisi-Zhang equation Phys. Rev. Lett. 78903

Lässig M and Kinzelbach H 1998 Upper critical dimension of the Kardar-Parisi-Zhang equation Phys. Rev. Lett. 805459 (erratum)

Lässig M and Kinzelbach H 1998 Reply: upper critical dimension of the Kardar-Parisi-Zhang equation Phys. Rev. Lett. 80889

[16] Bouchaud J P and Cates M E 1993 Self-consistent approach the Kardar-Parisi-Zhang equation Phys. Rev. E 47 R1455

Bouchaud J P and Cates M E 1993 Self-consistent approach the Kardar-Parisi-Zhang equation Phys. Rev. E 48635 (erratum)

[17] Bhattacharjee J K 1998 Upper critical dimension of the Kardar-Parisi-Zhang equation J. Phys. A 31 L93

[18] Colaiori F and Moore M A 2001 Upper critical dimension, dynamic exponent, and scaling functions in the mode-coupling theory for the Kardar-Parisi-Zhang equation Phys. Rev. Lett. 863946

[19] Katzav E and Schwartz M 2002 Existence of the upper critical dimension of the Kardar-Parisi-Zhang equation Physica A 30969

[20] Fogedby H C 2006 Kardar-Parisi-Zhang equation in the weak noise limit: pattern formation and upper critical dimension Phys. Rev. E $\mathbf{7 3} 031104$

[21] Moore M A, Blum T, Doherty J P, Marsili M, Bouchaud J-P and Claudin P 1995 Glassy solutions of the Kardar-Parisi-Zhang equation Phys. Rev. Lett. 744257

[22] Castellano C, Marsili M and Pietronero L 1998 Nonperturbative renormalization of the Kardar-Parisi-Zhang growth dynamics Phys. Rev. Lett. 803527

[23] Castellano C, Gabrielli A, Marsili M, Muñoz M A and Pietronero L 1998 High dimensional behavior of the Kardar-Parisi-Zhang growth dynamics Phys. Rev. E 58 R5209(R)

[24] Castellano C, Marsili M, Muñoz M A and Pietronero L 1999 Scale invariant dynamics of surface growth Phys. Rev. E 596460

[25] Ala-Nissila T, Hjelt T, Kosterlitz J M and Venäläinen O 1993 Scaling exponents for kinetic roughening in higher dimensions J. Stat. Phys. 72207

[26] Marinari E, Pagnani A and Parisi G 2000 Critical exponents of the KPZ equation via multi-surface coding numerical simulations J. Phys. A 338181

[27] Marinari E, Pagnani A, Parisi G and Rácz Z 2002 Width distributions and the upper critical dimension of Kardar-Parisi-Zhang interfaces Phys. Rev. E 65026136

[28] Pagnani A and Parisi G 2013 Multisurface coding simulations of the restricted solid-on-solid model in four dimensions Phys. Rev. E 87010102

[29] Pagnani A and Parisi G 2015 Numerical estimate of the Kardar-Parisi-Zhang universality class in $(2+1)$ dimensions Phys. Rev. E 92010101

[30] Kim J M and Kim S-W 2013 Restricted solid-on-solid model with a proper restriction parameter $N$ in $4+1$ dimensions Phys. Rev. E 88034102

[31] Kim S-W and Kim J M 2014 A restricted solid-on-solid model in higher dimensions J. Stat. Mech. P07005

[32] Alves S G, Oliveira T J and Ferreira S C 2014 Universality of fluctuations in the Kardar-Parisi-Zhang class in high dimensions and its upper critical dimension Phys. Rev. E 90020103

[33] Rodrigues E A, Mello B A and Oliveira F A 2015 Growth exponents of the etching model in high dimensions J. Phys. A: Math. Theor. 48035001

[34] Alves S G and Ferreira S C 2016 Scaling, cumulant ratios, and height distribution of ballistic deposition in $3+1$ and $4+1$ dimensions Phys. Rev. E 93052131

[35] Kim J M 1998 Phase transition of the Kardar-Parisi-Zhang equation in four substrate dimensions Phys. Rev. Lett. 80888

[36] Schwartz M and Perlsman E 2012 Upper critical dimension of the Kardar-Parisi-Zhang equation Phys. Rev. E 85050103

[37] Wio H S, Escudero C, Revelli J A, Deza R R and de la Lama M S 2011 Recent developments on the KardarParisi-Zhang surface-growth equation Phil. Trans. R. Soc. A 369296

[38] Moser K, Kertész J and Wolf D E 1991 Numerical solution of the Kardar-Parisi-Zhang equation in one, two and three dimensions Physica A 178 215-26

[39] Buceta R C 2005 Generalized discretization of the Kardar-Parisi-Zhang equation Phys. Rev. E 72017701

[40] Tu Y 1992 Instability in a continuum kinetic-growth model with surface relaxation Phys. Rev. A 46 R729

[41] Kim J M and Das Sarma S 1994 Discrete models for conserved growth equations Phys. Rev. Lett. 722903

[42] Dasgupta C, Das Sarma S and Kim J M 1996 Controlled instability and multiscaling in models of epitaxial growth Phys. Rev. E 54 R4552(R) 
[43] Dasgupta C, Kim J M, Dutta M and Das Sarma S 1997 Instability, intermittency, and multiscaling in discrete growth models of kinetic roughening Phys. Rev. E 552235

[44] Sasamoto T and Spohn H 2010 One-dimensional Kardar-Parisi-Zhang equation: an exact solution and its universality Phys. Rev. Lett. 104230602

[45] Miranda V G and Aarão Reis F D A 2008 Numerical study of the Kardar-Parisi-Zhang equation Phys. Rev. E 77031134 